\title{
Versatility of advancement flaps for nasal reconstruction following Mohs` micrographic surgery*
}

\author{
Felipe Bochnia Cerci $^{1}$
}

DOI: http:/ / dx.doi.org/10.1590/abd1806-4841.20187284

\begin{abstract}
Advancement flaps are important reconstructive options after skin cancer removal on the nose. Donor areas vary according to defect location and size. The objective of this article is to illustrate the versatility of advancement flaps in nasal reconstruction. Five patients were selected. All cases were treated with Mohs' micrographic surgery prior to reconstruction to ensure that $100 \%$ of the surgical margins were free of cancer. Advancement flaps can be used to repair a wide variety of surgical defects on the nose with good matching of skin color, texture, and thickness. With careful planning, resulting scars can be camouflaged in natural boundary lines, leading to good functional and cosmetic outcomes.
\end{abstract}

Keywords: Mohs' surgery; Nose neoplasms; Surgical flaps

\section{INTRODUCTION}

Advancement flaps are important reconstructive options after skin cancer removal. On the nose, these flaps are mainly used for defects located on the superior two-thirds of the nose. ${ }^{1,2}$ However, distal defects may also be repaired with advancement flaps. ${ }^{3}$ Donor areas vary according to defect location and consist of nasal subunits, such as nasal sidewalls, tip and dorsum, as well as adjacent facial cosmetic units such as medial cheeks, glabella and forehead. ${ }^{1,2}$

Since the nose is one of the areas most affected by non-melanoma skin cancer, it is essential that dermatologic surgeons become familiarized with reconstructive options for this site. Therefore, the objective of this article is to illustrate the versatility of some of these flaps in nasal reconstruction.

\section{CASE REPORTS}

Five patients were selected to illustrate the flaps. All cases were treated with Mohs' micrographic surgery prior to reconstruction to ensure that $100 \%$ of the surgical margins were free of cancer. Mohs surgeries and reconstructions were performed under local anesthesia with bupivacaine and lidocaine, and epinephrine. All flaps were closed in standard layered fashion with subdermal absorbable and cutaneous nonabsorbable sutures. Suture removal was performed one week postoperatively.

\section{Case 1}

A 68-year-old woman presented with a $2 \times 1 \mathrm{~cm}$ recurrent morpheaform basal cell carcinoma (BCC) on the left nasal sidewall. The tumor had been treated three years earlier in another institution by regular excision. After five stages of Mohs' surgery, the resulting defect measured $3.4 \times 3.2 \mathrm{~cm}$ and affected the left nasal sidewall and nasal dorsum. She was repaired with a crescentic advancement flap that recruited skin from the cheek (Figure 1).

Key factors in choice: off-center defect; utilizes laxity of medial cheek and nasal sidewall; unilateral crescentic advancement flap hides most incision lines in natural folds and subunit junctions.

\footnotetext{
Received 11 June 2017.

Accepted 03 November 2017.

* Work conducted at the Dermatology Unit, Hospital Santa Casa de Curitiba, Curitiba (PR), Brasil.

Financial support: None.

Conflict of interest: None.

1 Dermatology Unit, Hospital de Clínicas, Universidade Federal do Paraná, Curitiba (PR), Brasil.
}

MAILING AdDREss:

Felipe Bochnia Cerci

E-mail: cercihc@hotmail.com 
Case 2

A 70-year-old woman presented with a nodular BCC on the nasal dorsum. The resulting defect after one stage of Mohs' surgery measured $2 \times 1.6 \mathrm{~cm}$. Patient was repaired with a single advancement flap that recruited skin from the upper nose, glabella and forehead (Figure 2).

Key factors in choice: midline defect restricted to the nasal dorsum; laxity of the glabella and inferior forehead; vertical incision lines camouflaged between nasal dorsum and sidewalls; horizontal incision lines camouflaged above the brows.

\section{Case 3}

A 71-year-old woman presented with a nodular BCC on the upper lateral portion of the nasal tip. The resulting defect after one stage of Mohs surgery measured $1 \times 0.9 \mathrm{~cm}$. She was repaired with a Burow's triangle flap, known in this location as an "east-west" flap, that recruited skin from the nasal tip and dorsum (Figure 3).
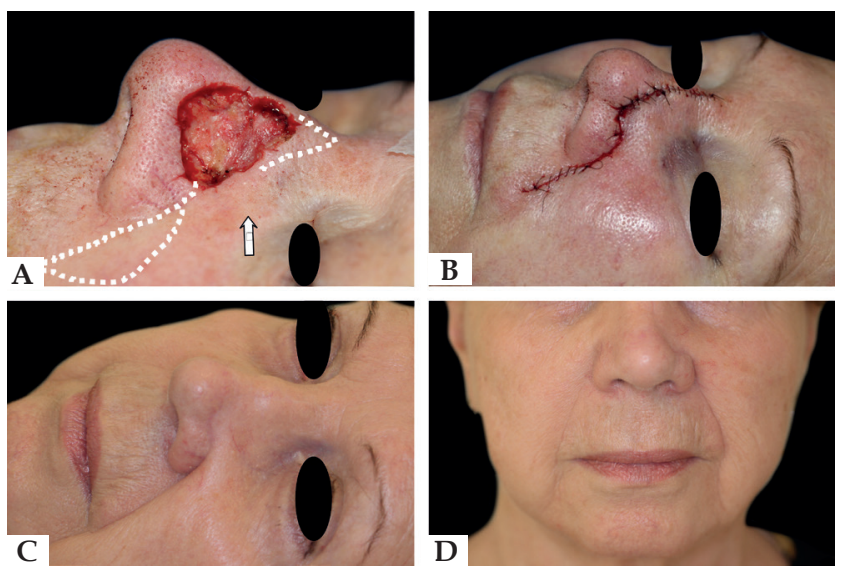

Figure 1: A - Surgical defect. Flap design (dotted line). The arrow indicates the flap movement, B - Immediate postoperative C Lateral view, result at 18 months, D - Frontal view with camouflaged incisions
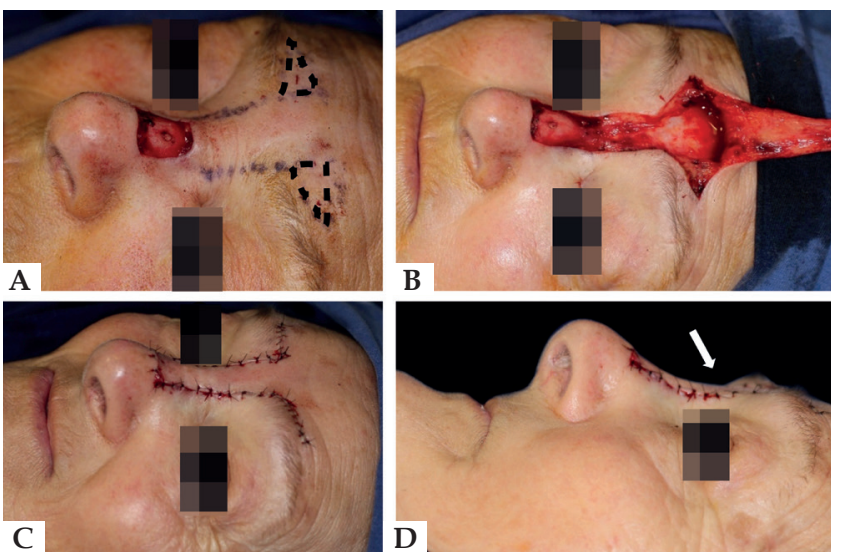

Figure 2: A - Surgical defect. Flap design with the standing cones demarcated above the brows, B - Flap raised, C - Immediate postoperative, D - Tacking suture on the superior portion of the nasal dorsum to recreate nasal root concavity (white arrow)
Key factors in choice: off-center small defect; broad nasal tip; primary closure would have affected right ala.

Case 4

A 42-year-old man presented with an invasive squamous cell carcinoma on the right nasal sidewall and nasal dorsum. The resulting defect after one stage of Mohs measured $2.5 \times 2.2 \mathrm{~cm}$. He was repaired with a bilateral advancement flap that recruited skin from the nose and cheek (Figures 4 and 5).

Key factors in choice: large off-center size defect; utilizes laxity of medial cheek and nose; insuf $\square$ cient laxity for single advancement flap; incision lines camouflaged in natural folds and subunit.

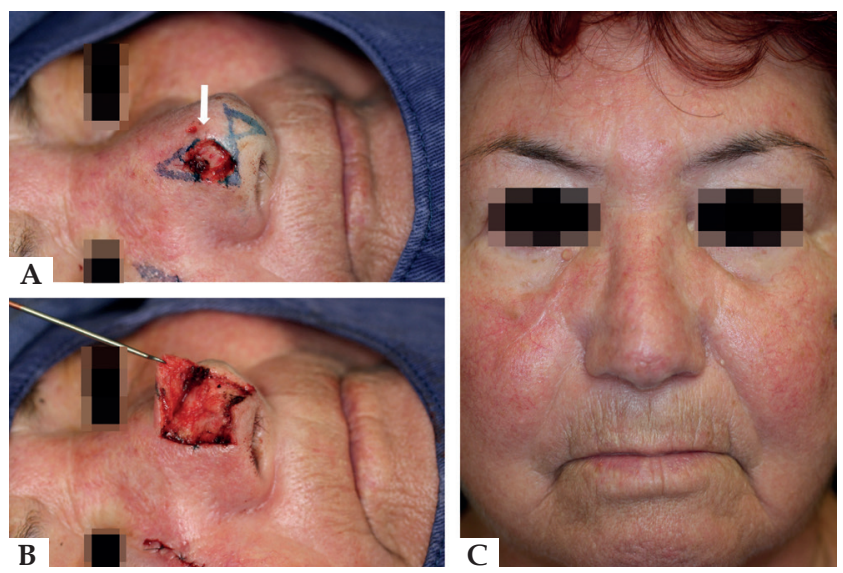

Figure 3: A - Surgical defect with the "east-west" flap design. The white arrow indicates the flap movement, B - Flap undermined on the supraperichondrial plane, C - 2-month postoperative
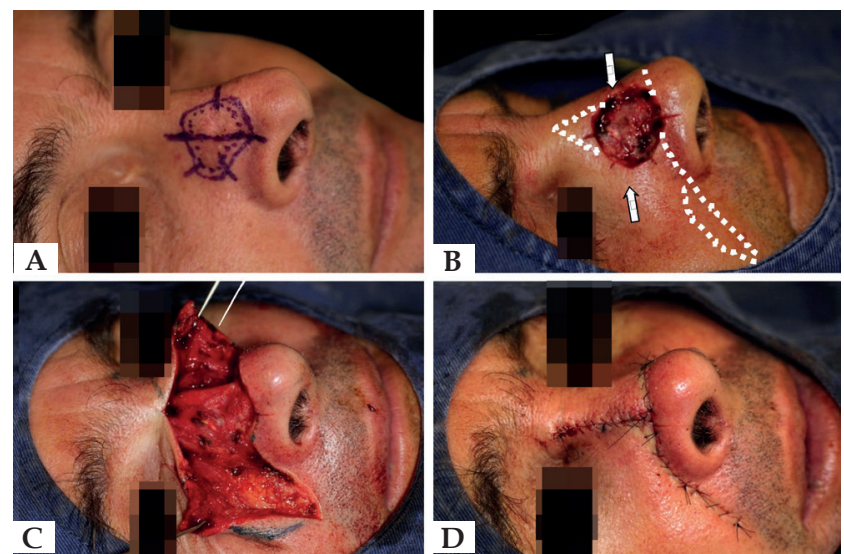

FIGURE 4: A - Invasive squamous cell carcinoma marked for Mohs' surgery. B - Resulting defect with the flap design (dotted line). Arrows indicate bilateral advancement to be performed C - Flap elevated, D - Immediate postoperative 

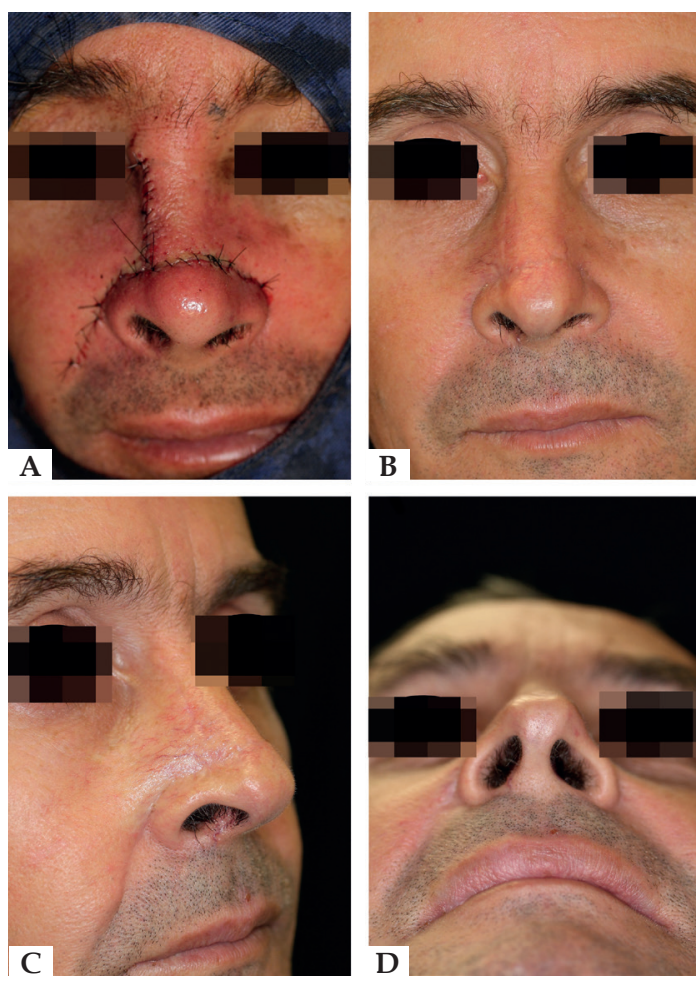

Figure 5: A - Suture lines placed on natural sulcus and on the boundaries between the nasal subunits. Vertical incision: right nasal sidewall and nasal dorsum. Horizontal incision: nasolabial fold; alar groove; nasal tip and nasal dorsum, B - Frontal view, 7-month postoperative, C - Oblique view, D - Inferior view

\section{Case 5}

A 54-year-old woman presented with a nodular BCC on the nasal tip. The resulting defect after one stage of Mohs measured 1.5 $x 1 \mathrm{~cm}$ and affected the nasal tip and a small area of the nasal dorsum. She was repaired with a VY nasalis sling flap that recruited skin from the nasal dorsum (Figure 6).

Key factors in choice: off-center upper nasal tip defect; good flap mobility; well-developed nasalis muscle pedicle for $\square$ ap vascularity.

\section{DISCUSSION}

Nasal reconstruction is often a challenge because of the multiple concavities and convexities over the nasal surface. Several approaches may be considered for nasal repair: healing by second intention, primary closure, grafts, $\square$ aps or combined methods. ${ }^{1-7}$ Various factors may contribute to the decision, including patient input, skin laxity, color, texture, sebaceous quality, defect size and depth, and subunits involved. ${ }^{8}$ In the present cases, other reconstructive options were considered and would also have led to good outcomes.

Several factors should be taken into account when considering an advancement flap to repair a nasal defect. First, the surgeon should determine whether there is an adequate tissue reservoir in the flap donor area. This may be determined by using fingers to
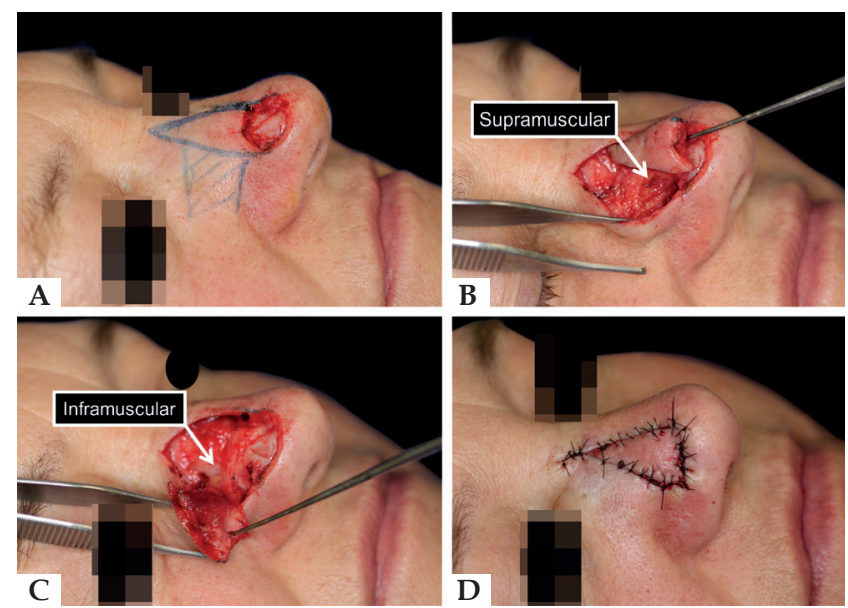

Figure 6: A - Surgical defect. Flap design (continuous line). The rectangle on the nasal sidewall indicates the myocutaneous pedicle, B and C - Bilevel undermining on two planes: infra and supramuscular, until the nasofacial sulcus D - Immediate postoperative

pinch the skin in various directions. The surgeon should also endeavor to make incisions such that the final suture lines are along skin tension lines, borders of cosmetic units, and/or rhytides. ${ }^{9}$

The cases presented here demonstrate some key points: In cases 1 and 4, care must be taken when undermining beyond the nasofacial sulcus to avoid damage of deeper structures. On the nose, undermining is supraperichondrial, whereas on the cheek, it is in the subcutaneous tissue. For adequate flap movement, and to avoid ala distortion, a large standing cone on the nasolabial sulcus must be resected. ${ }^{8}$

In case 2, the patient must have enough laxity on the glabella and forehead to prevent an unacceptable degree of nasal tip elevation. On the upper part of the nose, a tacking suture is essential to avoid a straightened glabellar angle. ${ }^{10}$ In case 3, patients with a thin nasal tip are not good candidates for the "east-west" flap. Careful undermining is important to avoid damaging the cartilages. ${ }^{3,4}$ In case 5, the flap must have good mobility to avoid pulling the alar rim or nasal tip. To prevent trapdoor, the defect may be deepened to better fit the flap, if necessary. ${ }^{5}$

As demonstrated, advancement flaps can be used to repair a wide variety of surgical defects on the nose, with good matching of skin color, texture, and thickness. Furthermore, with careful planning, the resulting scars can be camouflaged in natural boundary lines, leading to good functional and cosmetic outcomes. $\square$ 


\section{REFERENCES}

1. Salmon PJ. Repair of the nasal sidewall. Br J Dermatol. 2014;171:17-22.

2. Vinciullo C. Reconstructing the nasal dorsum. Br J Dermatol. 2014;171:7-16.

3. Lambert RW, Dzubow LM. A dorsal nasal advancement flap for off-midline defects. J Am Acad Dermatol. 2004;50:380-3.

4. Goldberg LH, Alam M. Horizontal advancement flap for symmetric reconstruction of small to medium-sized cutaneous defects of the lateral nasal supratip. J Am Acad Dermatol. 2003;49:685-9.

5. Willey A, Papadopoulos DJ, Swanson NA, Lee KK. Modified single-sling myocutaneous island pedicle flap: series of 61 reconstructions. Dermatol Surg. 2008:34:1527-35.
6. Yoo SS, Miller SJ. The crescentic advancement flap revisited. Dermatol Surg. 2003;29:856-8.

7. Cerci FB, Nguyen TH. Paramedian forehead flap for complex nasal defects following Mohs micrographic surgery. Surg Cosmet Dermatol. 2014;6:17-24.

8. Haugen TW, Frodel JL. Reconstruction of complex nasal dorsal and sidewall defects: is the nasal sidewall subunit necessary? Arch Facial Plast Surg. 2011:13:343-6.

9. Krishnan R, Garman M, Nunez-Gussman J, Orengo I. Advancement flaps: a basic theme with many variations. Dermatol Surg. 2005;31:986-94.

10. Rintala AE, Asko-Seljavaara S.Reconstruction of midline skin defects of the nose. Scand J Plast Reconstr Surg. 1969;3:105-8. 